\title{
Aufsätze
}

\section{Michael Höhne}

\section{Die Reform der vorsätzlichen Tötungsdelikte}

\section{Warum ist sie bisher gescheitert und wie könnte sie aussehen?}

Aus der juristischen Literatur ist eine Auseinandersetzung mit der Reform der vorsätzlichen Tötungsdelikte nicht mehr wegzudenken. Die Thematik hat durch die Äußerungen der schleswig-holsteinischen Justizministerin Anke Spoorendonk bei der Justizministerkonferenz am 14.11.2013 in Berlin zur Notwendigkeit einer Reform aufgrund der nationalsozialistischen Herkunft der Normen über vorsätzliche Tötungsdelikte und den daran anknüpfenden Gesetzantrag ${ }^{1}$ erneut an Aktualität gewonnen. ${ }^{2}$ Die Debatte hat wieder Eingang in die politischen Ebenen gefunden. Nunmehr wurde sogar von Bundesjustizminister Heiko Maas eine Expertengruppe zur Überarbeitung der Tötungsdelikte einberufen, die am 20.5.2014 ihre Arbeit aufgenommen hat und im Laufe des nächsten Jahres einen Reformvorschlag ausgearbeitet haben soll. ${ }^{3}$ Gleichwohl stellt sich die Frage, warum trotz der - scheinbar offensichtlichen - Reformbedürftigkeit eine Reform bisher ausblieb.

\section{Hintergründe}

„Das gegenwärtige System der Tötungsdelikte bedarf einer Reform [...]“, bekannte die Bundesregierung bereits im Jahre 1996 im Rahmen einer Antwort auf eine Kleine Anfrage. ${ }^{4}$ Die auch aus Sicht des Bundesrates notwendige Reform ${ }^{5}$ sollte dabei auch die Abgrenzung von Mord und Totschlag umfassen. ${ }^{6}$ Somit haben zwei Organe, die das öffentliche Gesetzgebungsverfahren einleiten können (Art. 76 Abs. 1 GG), schon einmal die Reformbedürftigkeit anerkannt. Umso erstaunlicher ist es, dass immer noch keine neue Regelung zur Abgrenzung von Mord und Totschlag gefunden wurde. Am 21.3.2001 gab es ersichtlich einen

1 Siehe BR-Drucks. 54/14, Gesetzantrag des Landes Schleswig-Holstein: Entwurf eines ... Gesetzes zur Änderung des Strafgesetzbuches - Bereinigung der $\$ \$ 211 \mathrm{ff}$. (... StRÄndG).

$2 \mathrm{Zu}$ diesen Entwicklungen aus der Sicht der Initiatorin: Spoorendonk, Die schleswig-holsteinische Bundesratsinitiative für eine Bereinigung der $\$ \$ 211 \mathrm{ff}$. Strafgesetzbuch, SchlHA 2014, $77 \mathrm{ff}$.

3 Maas, Warum wir endlich eine Reform der Tötungsdelikte brauchen, RuP 2014, 65, 67.

4 BT-Drucks. 13/4830, 7 (Antwort der Bundesregierung auf die Kleine Anfrage der Abgeordneten Volker Beck [Köln], Amke Dietert-Scheuer, Gerald Häfner, weiterer Abgeordneter und der Fraktion BÜNDNIS 90/DIE GRÜNEN - Drucksache 13/4705 - Vollzug der lebenslangen Freiheitsstrafe und Reform der Tötungsdelikte im Strafgesetzbuch).

5 Vgl. BT-Drucks. 13/8587, 55 (Stellungnahme zum 6. StrRG).

6 BT-Drucks. 13/4830, 8 (Fn. 4); mit Hervorhebung, dass dies insbesondere das Reformbedürftige sei: BTDrucks. 13/8587, 78 (Gegenäußerung der Bundesregierung zum $6_{4}$ StrRG). 
nicht weiter verfolgt wurde.

Die wissenschaftliche Diskussion beschränkt sich größtenteils darauf, die aktuelle Lage zu analysieren, zu kritisieren und eine bessere Abgrenzungsmöglichkeit für die Zukunft zu suchen. Die Frage, warum der Gesetzgeber nicht tätig wurde und nicht wird, spielt dabei eine sehr untergeordnete Rolle. ${ }^{8}$ Es muss aber Gründe geben. Eine diesbezügliche Ursachenforschung könnte eine Reform unterstützen, denn eventuell ist es möglich, bestehende Hindernisse zu beseitigen oder zu umgehen.

\section{Die Reformbedürftigkeit}

Die Reformbedürftigkeit zeigt sich insbesondere anhand von dreierlei Problemkreisen: Erstens hat sich die tatbestandliche Ausgestaltung des $\$ 211$ StGB seit 1941 nicht mehr geändert ${ }^{9}$ und geht damit auf die nationalsozialistische Rechtsordnung zurück. Dabei wird insbesondere als problematisch angesehen, dass \211 StGB auf den Mörder abstellt und damit deutlich seine nationalsozialistische Herkunft offenbart, ${ }^{10}$ wobei auch manchen Mordmerkmalen eine „braune Färbung "11 nachgesagt wird.

Zweitens gründet das Problem der Abgrenzung zwischen Mord und Totschlag darin, dass die Mordmerkmale nicht geeignet sind, eine klare Linie zu ziehen. So sind beispielsweise die sonstigen niedrigen Beweggründe zu unbestimmt, was zu Auslegungsschwierigkeiten führt. Dabei verdeutlicht sich, dass den Mordmerkmalen kein einheitliches Leitprinzip zugrunde liegt. Auch ist die Rigidität mancher Mordmerkmale (z.B. Heimtücke) problematisch. Entlastende Aspekte werden nicht berücksichtigt, obwohl diese teilweise den Mordmerkmalen immanent sind. Dadurch kann aber der Sanktionensprung durch die absolute Strafandrohung bei Mord schwerlich gerechtfertigt werden. Des Weiteren ist eine Steigerung des Unwertgehalts in Bezug auf die Zerstörung des Rechtsguts Leben kaum möglich, was eine „hoffnungslose Überschätzung“ der Steigerung von Unrecht bzw. Schuld bei bestimmten Mordmerkmalen (wie etwa bei Habgier) herbeiführt

7 Mangels offizieller Stellungnahmen ergeben sich (dürre) Informationen nur aus der Erwähnung in wissenschaftlichen Veröffentlichungen und in öffentlichen Medien, wobei sogar der formale Status dieses Entwurfs unterschiedlich bezeichnet wird: „Eckpunktepapier“, „Arbeitsentwurf“, „Reformentwurf“; vgl. Schnorr/Wissing, Strafrechtsänderungsgesetz, ZRP 2001, 391 f.; Otto, Neue Entwicklungen im Bereich der vorsätzlichen Tötungsdelikte, Jura 2003, 612, 621 f.; Sotelsek, Zur Quantifizierung von Unrecht und Schuld bei vorsätzlichen Tötungen, 2012, 263; DER SPIEGEL, Heft 25/2001, 17.

8 Allerdings warf Frommel konkret die Frage auf „Wieso gelingt es nicht, die allgemein für reformbedürftig angesehenen Tötungsdelikte zu novellieren?” (in: Weber [Hrsg.], Leben ohne Lebenslänglich, 1988, 69 ff.), bezog sich in ihrer Beantwortung aber fast nur auf Aspekte der Möglichkeit einer gelungenen Abgrenzung von Mord und Totschlag.

9 RGBl. I, 549 (Gesetz zur Änderung des Reichsstrafgesetzbuches vom 4.9.1941); Näheres zur Entwicklung bei Thomas, Die Geschichte des Mordparagraphen, 1985, 5 ff.; Linka, Mord und Totschlag (SS 211-213 StGB), 2008, 39 ff. Mit dem 3. StrÄndG vom 4.8.1953 (BGBl. I, 735) wurde die Todesstrafe in $\$ 211$ StGB durch die lebenslange Zuchthausstrafe ersetzt (seit 25.6.1969 lebenslange Freiheitsstrafe, BGBl. I, 645 - 1. StrRG), wodurch auch Abs. 3 hinfällig wurde, der in Ausnahmefällen statt Todesstrafe die lebenslange Haft ermöglichte.

10 In der Formulierung spiegelt sich die Tätertypenlehre wider; vgl. Hirsch, in: Bernsmann/Fischer (Hrsg.), Festschr. f. Rissing-van Saan, 2011, 219 ff., 222; ausführlich dazu: Kubik/Zimmermann, Mord und/oder Totschlag?, StV 2013, 582.

11 Gössel, siehe dazu Pintaske/Sitzer, Die Erarbeitung gemeinsamer Grundlagen zum systematischen Verhältnis von Mord und Totschlag und zur Reform der Tötungsdelikte (Diskussionsbericht), in: Jahn/ Nack (Hrsg.), Rechtsprechung, Gesetzgebung, Lehre, 2010, 61 ff., 62; kritisch aus der aktuellen Literatur auch König, Überlegungen zur Reform der Tötungsdelikts-Normen ${ }_{8}$ RuP 2014, 9. 
und somit von einer Unterbewertung des Rechtsguts Leben gesprochen werden

kann. ${ }^{12}$

Endlich (drittens) ist die Systematik des Gesetzes nicht eindeutig, was sich auf das Verhältnis von Mord und Totschlag auswirkt. Dadurch, dass die Rechtsprechung davon ausgeht, dass Mord und Totschlag zwei selbstständige Delikte sind $^{13}$ und Mord somit keine Qualifikation des Totschlags darstellt, ${ }^{14}$ findet im Bereich der Teilnahme bei täterbezogenen Merkmalen $\$ 28$ Abs. 1 StGB Anwendung, ${ }^{15}$ wobei sich dann Probleme ergeben, die von der Rechtsprechung unter Strafzumessungsgesichtspunkten angemessen gelöst werden, ${ }^{16}$ jedoch teilweise über dogmatisch fragwürdige Konstruktionen. ${ }^{17}$

\section{Mögliche Gründe für das Ausbleiben der Reform}

\section{Die statistisch geringe Bedeutung der Tötungsdelikte}

Man könnte zunächst zwar nicht die Reformbedürftigkeit, aber eventuell die Dringlichkeit der Reform aufgrund der Seltenheit der vorsätzlichen Tötungsdelikte im Vergleich zu anderen Delikten verneinen. ${ }^{18} 2013$ wurden 5.961.662 Straftaten erfasst, worunter sich nur 814 Morde (282 vollendet) und 1.736 Fälle von Totschlag (303 vollendet) befanden. ${ }^{19}$ Mord und Totschlag machen damit zwar nur $0,0428 \%$ der erfassten Fälle aus. Sie nehmen in der öffentlichen Wahrnehmung aber einen ungleich höheren Stellenwert ein, weil die vorsätzliche Tötung der „Bruch eines zentralen gesellschaftlichen Tabus ${ }^{\text {"20 }}$ ist. Die statistische Bedeutungsarmut der vorsätzlichen Tötungsdelikte dürfte daher für ein Ausbleiben der Reform nicht verantwortlich sein.

\section{Die Erträglichkeit der aktuellen Situation}

Die dargestellte Reformbedürftigkeit bezieht sich auf die gesetzliche Regelung von Mord und Totschlag. Die Ergebnisse der Rechtsprechung bei der Beurteilung vorsätzlicher Tötungsdelikte werden hingegen teilweise als so gelungen empfunden, dass keine Eilbedürftigkeit ${ }^{21}$ und sogar keine Notwendigkeit einer Reform ${ }^{22}$ angenommen wird. stände des Mordes, des Totschlags und der Kindestötung ( $\mathbb{S} 211$ bis 213, 217 StGB) neu abzugrenzen?, Gutachten D zum 53. DJT (1980), 36 ff.

13 Siehe nur BGHSt 1, 368, $370 \mathrm{ff}$.

14 Zur herrschenden Ansicht im Schrifttum, die dagegen den Totschlag als Grunddelikt und den Mord als Qualifikation sieht, statt aller NK-Neumann, 4. Aufl. (2013), Vor \$211 StGB Rn. 154.

15 BGHSt 1, 368; BGH, StV 1989, 150; BGH, NStZ-RR 2002, 139.

16 Insbesondere Mittäterschaft bei getrennter Verwirklichung von $\$ 211$ StGB und $\$ 212$ StGB durch mehrere Täter, die sog. gekreuzten Mordmerkmale und die „Sperrwirkung“; Rissing-van Saan, in: Jahn/ Nack (Hrsg.), Rechtsprechung, Gesetzgebung, Lehre, 2010, 26 ff., 36 ff.

17 Gropp, Mord und Totschlag, in: Schneider et al. (Hrsg.), Festschr. f. Seebode, 2008, 125 ff., 138.

18 Vgl. Grünewald, Zur Abgrenzung von Mord und Totschlag - oder: Die vergessene Reform, JA 2012, 401.

19 Polizeiliche Kriminalstatistik 2013, vgl. http://www.bka.de/SharedDocs/Downloads/DE/Publikationen/PolizeilicheKriminalstatistik/2013/pks2013ImkBericht,templateId=raw,property=publicationFile.pdf//pks2013ImkBericht.pdf (abgerufen am 20.6.2014).

20 Egg, Vorwort, in: ders. (Hrsg.), Tötungsdelikte, 2002, 5 f., 5.

21 Lackner, Referat M zum 53. DJT (1980), 25 ff., 25.

22 Rebmann, siehe dazu Verhandlungen des 53. DJT (1980), Bd. II, M 52; aus der aktuellen Diskussion Bausback, FOCUS Magazin Nr. 24, 6.6.2014, 37 
Die Rechtsprechung hat Wege gefunden, einige der sich aus der aktuellen Gesetzeslage ergebenden Probleme abzumildern. Insbesondere die Absolutheit der lebenslangen Freiheitsstrafe hat deutliche Einschränkungen erfahren.

Auf der Tatbestandsebene existiert als Abmilderungsmechanismus zunächst die Verneinung des Vorliegens bedingt vorsätzlichen Handelns. Dabei dient die „Hemmschwellentheorie“ ${ }^{23}$ der Umgehung der Höchststrafe. ${ }^{24}$ Eine derartige Hemmschwelle wurde jedoch nie empirisch nachgewiesen oder psychologisch begründet. ${ }^{25}$ Kritisiert wird auch, dass revisionsrechtliche Beanstandungen der tatgerichtlichen Feststellungen zum Tötungsvorsatz sehr unterschiedlich ausfallen, die Urteile scheinbar ergebnisorientiert erarbeitet werden und somit eine unkalkulierbare Rechtsprechung ohne Rechtssicherheit besteht. ${ }^{26}$ Des Weiteren sind die restriktiven Auslegungen von Mordmerkmalen oftmals nicht überzeugend, wie etwa Subjektivierungen, ${ }^{27}$ denen eine teleologische Fundierung fehlt. ${ }^{28}$ Viele kontroverse Entscheidungen führen $\mathrm{zu}$ einer unübersichtlichen (höchstrichterlichen) Rechtsprechung, ${ }^{29}$ was eine unterschiedliche Restriktion der Mordmerkmale je nach Gericht und Richter begünstigt. ${ }^{30}$

Auf der Rechtsfolgenebene wird durch die wohl umstrittenste Umgehungsstrategie, der sog. Rechtsfolgenlösung des Großen Senats des Bundesgerichtshofs, ${ }^{31}$ bei tatbestandlich gegebener Heimtücke und gleichzeitigem Vorliegen außergewöhnlicher Umstände $₫ 49$ Abs. 1 Nr. 1 StGB angewandt und keine lebenslange Freiheitsstrafe verhängt. Zwar können so einzelfallgerechte Urteile ergehen, aber das Durchbrechen der absoluten Strafandrohung wird zu Recht als Rechtsanwendung contra legem und nicht praeter legem angesehen. ${ }^{32}$

Durch die Einführung von $\int 57 \mathrm{a}$ StGB ${ }^{33}$ der die Möglichkeit einer Entlassung bei lebenslanger Haft normiert, wurde die Problematik der Abgrenzung von Mord und Totschlag 1981 auf der Vollzugsebene entschärft. Der Verurteilte kann nach 15 Jahren Mindestverbüßungszeit entlassen werden ( $\int 57$ a Abs. 1 Nr. 1 StGB). Der Sanktionensprung ist dadurch weit weniger groß. Innerhalb der Normanwendung treten aber wiederum Probleme auf, die einer uneingeschränkt positiven Einschätzung das Fundament entziehen. So ist beispielsweise problematisch, dass der Begriff der besonderen Schwere der Schuld ( $\$ 57$ a Abs. 1 Nr. 2

Wegen einer besonders hohen Hemmschwelle zur Tötung eines Menschen kann auch bei größter Gefährlichkeit die Indizwirkung für Vorsatz im Rahmen der Gesamtwürdigung aller Tatumstände und der Täterpersönlichkeit entfallen; BGH, NStZ 1994, 585; BGH, StV 2004, 74, 75; siehe zu aktuellen Entwicklungen in der Rechtsprechung diesbezüglich Puppe, Tötungsvorsatz und Affekt, NStZ 2014, 183 ff. Geppert, Zur Abgrenzung von Vorsatz und Fahrlässigkeit, insbesondere bei Tötungsdelikten, Jura 2001, 55, 59; Trück, Die Problematik der Rechtsprechung des BGH zum bedingten Tötungsvorsatz, NStZ 2005, 233, 240.

25 Trück (Fn. 24), NStZ 2005, 233, 234; Geppert (Fn. 24), Jura 2001, 55, 59.

26 Verrel, (Noch kein) Ende der Hemmschwellentheorie?, NStZ 2004, 309, 310; Trück (Fn. 24), NStZ 2005, 233.

27 Z.B. hinsichtlich des Ausnutzungsbewusstseins bei Heimtücke oder der unbarmherzigen Gesinnung bei Grausamkeit, Schneider, in: MünchKommStGB, 2. Aufl. (2012), \$211 Rn. 29.

28 Schneider (Fn. 27), \$211Rn. 29.

29 Roxin, Zur normativen Einschränkung des Heimtückemerkmals bei Mord, in: Schöch et al. (Hrsg.), Festschr.f. Widmaier, 2008, 741 ff., 741; zur Heimtücke: Mitsch, Die Verfassungswidrigkeit des $\ 211$ StGB, JZ 2008, 336, 337 („Rutschbahn in immer tiefere Konfusion“).

30 Grünewald (Fn. 18), JA 2012, 401, 402; NK-Dünkel, 4. Aufl. (2013), §57a StGB Rn. 61.

31 BGHSt 30, 105.

32 Bruns, Richterliche Rechtsfortbildung oder unzulässige Gesetzesänderung der Strafdrohung für Mord?, JR 1981, 358, 362; Mitsch (Fn. 29), JZ 2008, 336, 337.

33 BGBl. I, 1329 (20. StrÄndG vom 15 12.1981). 
StGB) sehr unbestimmt ist ${ }^{34}$ und auch durch höchstrichterliche Rechtsprechung $^{35}$ nicht hinreichend konkretisiert wird. ${ }^{36}$

\section{b) Gedanken zur Akzeptanz des Empfindens einer erträglichen Praxissituation als Grund mangelnder Reformbereitschaft}

Die Rechtsprechung muss Abmilderungsmechanismen anwenden, um zu gerechten Urteilen zu kommen, und ist dabei immer heftiger Kritik ausgesetzt. Es kann zwar ein recht hohes Maß an Einzelfallgerechtigkeit vermutet werden, aber die im Grundgesetz verankerte Bindung an das Gesetz (Art. 20 Abs. 3 GG) und Dogmatik scheinen dabei nicht die höchste Priorität zu besitzen. ${ }^{37}$ Durch die Rechtsprechung sollen nur Probleme der Aktualität oder Abstraktion des Gesetzestextes ausgeglichen werden. Die gesetzliche Regelung weist aber noch weitere Probleme auf, die aufgrund der Gewaltenteilung vom Gesetzgeber und nicht von der Rechtsprechung beseitigt werden müssen. Dies führt dazu, dass die Strategien der Rechtsprechung „Spuren an dem Gebäude des rechtsstaatlichen Strafrechts zurücklassen "38.

Gleichzeitig ist es schwer, eine Aussage über „die Rechtsprechung“ zu tätigen, da meist nur die Urteile des Bundesgerichtshofs bewertet werden, in der Annahme, dass die Instanzgerichte sich daran ausrichten. Selbst wenn man alle eine lebenslange Freiheitsstrafe verhängenden Urteile untersuchte und zu dem Ergebnis käme, dass die verhängte Strafe nicht übermäßig war, ${ }^{39}$ würde dies keinen Aufschluss darüber bieten, ob auch in Fällen der Verhängung einer nur zeitigen Freiheitsstrafe bei vorsätzlicher Tötung die entsprechenden Urteile angemessen ausfielen.

\section{c) Annabmen zur Sicht der Legislative}

Der Vorstoß des Bundesjustizministeriums im Jahre $2001 \mathrm{zu}$ einer Reform beschränkte sich in Bezug auf die Abgrenzung von Mord und Totschlag darauf, die Rechtsfolgenlösung zu kodifizieren. ${ }^{40}$ Dies zeigt, dass dem Weg der Judikative grundsätzlich Vertrauen entgegengebracht wird, was als ein Kernpunkt des nicht hinreichenden Betreibens einer Reform zu vermuten ist. ${ }^{41}$ Würde die Rechtsprechung keine Wege finden, zu angemessenen Urteilen zu kommen, könnten und würden ungerechte Urteile an die Medien getragen. Es würde eher ein öffentlicher Druck auf den Gesetzgeber entstehen. Aspekte der Erschütterung des Rechtsstaats durch Umgehungsstrategien lassen sich aber schlechter instrumentalisieren und in der Öffentlichkeit wirksam äußern.

Steinhilber, Mord und Lebenslang, 2012, 176; Baltzer, 1998: Das Jahr der Wende in der Kriminalpolitik - auch im Hinblick auf die Verhängung und Vollstreckung lebenslanger Freiheitsstrafen?, StV 2010, 602,604 .

35 BGH, NJW 1995, 407.

36 Heine, Mord und Mordstrafe: Grundmängel der deutschen Konzeption und rechtsvergleichende Reformüberlegungen, GA 2000, 305, 309; Hoffmann-Holland, Besondere Schwere der Schuld i.S.d. \$57a StGB, StraFo 2006, 277.

37 Arzt/Weber/Heinrich/Hilgendorf, Strafrecht BT, 2. Aufl. (2009), 32.

38 Neumann, Zur (Nicht-)Reform der lebenslangen Freiheitsstrafe, in: Institut für Kriminalwissenschaften und Rechtsphilosophie Frankfurt am Main (Hrsg.), Irrwege der Strafgesetzgebung, 1999, 389 ff., 390.

39 So Staiger, Zu den Auswirkungen der tatbestandlichen Ausgestaltung des $\$ 211$ StGB, in: Jescheck/ Triffterer (Hrsg.), Ist die lebenslange Freiheitsstrafe verfassungswidrig?, 1978, 181 ff., 188, für den Zeitraum 1945-1977; ein Beispiel zu hoher Strafe stellt wohl der Fall des Kannibalen von Rotenburg dar, der eine lebenslange Freiheitsstrafe trotz einer Einwilligung des Opfers zur Tötung erhielt; Roxin, Das systematische Verhältnis von Mord und Totschlag, in: Jahn/Nack (Hrsg.), Rechtsprechung, Gesetzgebung, Lehre, 2010, $21 \mathrm{ff} ., 23$. 
Schon 1875 wurde resigniert festgestellt, „daß es keiner Rechtswissenschaft und keiner Gesetzgebung der Welt bis jetzt annähernd gelungen ist, ein rechtlich brauchbares Merkmal zu finden, wonach die schwersten (vermeintlich) todeswürdigen Tödtungen von den nächst-schwereren, nicht mehr todeswürdigen, unterschieden werden könnten. " 42 Zwar gibt es aus deutscher Sicht keine todeswürdigen Tötungen mehr, aber das Gesetz sieht Tötungen vor, bei denen eine lebenslange Strafe tatschuldangemessen sein soll. Wenn der Gesetzgeber keine zur allgemeinen Zustimmung geeignete Lösung finden kann, ist ein Ausharren nachvollziehbar, wenn auch nicht wünschenswert. Die Untätigkeit des Gesetzgebers kann also in der Unmöglichkeit der befriedigenden Abgrenzung von Mord und Totschlag begründet sein.

Unabhängig davon, wie viele Tatbestände für vorsätzliche Tötungen (ohne Sonderdelikte wie $\$ 216$ StGB) normiert werden, wird bei den meisten Reformvorschlägen grundsätzlich davon ausgegangen, dass eine Art „Durchschnittstötung“ existiert, also eine Tötung, die weder strafmildernde noch strafschärfende Kategorien verwirklicht. ${ }^{43}$ Bei Verwirklichung derartiger Kategorien liegt keine „Durchschnittstötung“ mehr vor, und es ist anders zu bestrafen. Dabei werden aber verschiedene Modelle verfolgt. Sowohl strafschärfende als auch strafmildernde Abstufungen können jeweils innerhalb eines Strafrahmens, unter Hinzunahme von besonderen Strafzumessungsregeln oder durch tatbestandliche Abstufungen herbeigeführt werden. Grob aufteilen lassen sich die Vorschläge anhand der Anzahl der zugrunde gelegten Tatbestände: Einheitstatbestand (ein Tatbestand mit entsprechend großem Strafrahmen), privilegierungs- und qualifizierungsbezogenes Zweistufenmodell (tatbestandliche Abgrenzung nur privilegierter oder nur qualifizierter Tötungen) und Dreistufenmodell (tatbestandliche Abgrenzungen sowohl privilegierter als auch qualifizierter Art). Überall dort, wo keine Tatbestandsabstufung zu einer Privilegierung oder Qualifizierung stattfindet, können dann entweder nur ein Strafrahmen oder zusätzlich Strafzumessungserwägungen normiert sein.

\section{a) Probleme der Formulierung von Abgrenzungsmerkmalen}

Es besteht bei der Frage, wie minder schwere Fälle inhaltlich von einer „Durchschnittstötung“ (tatbestandlich) abgegrenzt werden können, ein breiter Konsens für gewisse Grundstrukturen. Einerseits soll es sich strafmildernd auswirken, wenn sich der Täter aufgrund einer begreiflich heftigen Gemütsregung zu der Tat hat hinreißen lassen, ${ }^{44}$ andererseits, wenn Beweggründe vorliegen, die „achtenswert“ sind. ${ }^{45}$ Es wäre für den Gesetzgeber durchaus möglich, eine Privilevon Typusbegriffen schon an der Länge und Unübersichtlichkeit der Norm leidet, der Reformentwurf in Morris, Die normative Restriktion des Heimtückebegriffes auf Basis der Teilverwirklichung von Rechtfertigungsgründen, 2010, 203 und die Konzeption von Müssig, Mord und Totschlag, 2005, die nicht ausformuliert wurde, aber darunter leidet, dass in vielen Konstellationen eine andere Wertung im Vergleich zur lex lata entsteht (vgl. Sotelsek [Fn. 7], 59). Eine umfangreiche Auseinandersetzung erfahren die meisten deutschen Vorschläge der letzten gut 50 Jahre bei Sotelsek (Fn. 7), 175 ff. - ohne die Vorschläge in Beckmann, Die Reform der Tötungsdelikte, in: Börner/Fabricius-Brand (Hrsg.), 3. Alternativer Juristinnen- und Juristentag, 1994, 61 ff., 71 f.; Morris (s.o. [in dieser Fn.]), 203; Reizel, Der Mordtatbestand de lege ferenda, 2006, 240 f.; Fahlbusch, Über die Unhaltbarkeit des Zustandes der Mordmerkmale in $\$ 211$ StGB, 2008, 339; Steinhilber (Fn. 34), 257 f.; Kubik/Zimmermann (Fn. 10), StV 2013, 582, 588; Deckers/Fischer/König/Bernsmann, Zur Reform der Tötungsdelikte Mord und Totschlag, NStZ 2014, 9, 16.

44 Beispielsweise der Vorschlag von Fuhrmann, Referat M zum 53. DJT (1980), 7 ff. (7, 10 und 15). 
gierung zu schaffen, die auf breite Zustimmung stößt. Der privilegierungsbezo-

gene Teil einer Neuregelung kann demnach ein Zögern des Gesetzgebers kaum rechtfertigen.

Die Abgrenzung „schwerer wiegender“ Tötungen ist hingegen schwierig. Eine solche könnte anhand von Leitprinzipien, anhand einer Kasuistik oder durch eine Kombination daraus vorgenommen werden.

\section{a) Leitprinzipien}

Es existiert schon kein Leitprinzip, das vollends überzeugen kann. So stehen dem Verwerflichkeitskonzept ${ }^{46}$ (auch in Form eines Abstellens auf eine Zweck-Mittel-Relation), ${ }^{47}$ dem Gefährlichkeitskonzept ${ }^{48}$ und dem Prämeditationskonzept $^{49}$ gewichtige Kritikpunkte entgegen.

\section{bb) Problematik einer Kasuistik}

Fast alle Reformkonzepte legen eine Kasuistik für strafschärfende Aspekte zugrunde und wollen nicht allein eine Generalklausel normiert sehen. Gesetzestechnisch kann eine Kasuistik durch die Nennung von Regelbeispielen oder durch die Aufzählung von Qualifikationsmerkmalen eingebracht werden. Problematisch bei einer Kasuistik ist allgemein, dass diese für besonders schwere Tötungen aufgrund der Vielzahl der Begehungsmöglichkeiten unvollständig bleiben muss. ${ }^{50}$ Eine Ausgestaltung als Qualifikationsmerkmal trägt das Risiko in sich, dass in anderen, nicht genannten Fällen eine entsprechende strafrechtliche Handhabung nicht möglich ist. Bei beiden Regelungsgestaltungen ist zudem die Bandbreite vorgeschlagener Fälle groß,${ }^{51}$ was auch auf die unterschiedlichen, zugrunde gelegten Leitprinzipien zurückzuführen ist. Schon diese Unterschiedlichkeit deutet darauf hin, dass kaum klar ist, wie eine Kasuistik aussehen müsste. Aber auch Fälle, die häufig genannt werden (z.B. Verdeckung einer Straftat), ${ }^{52}$ erfahren deutliche Kritik. ${ }^{53}$

Es ist somit bereits unklar, wie eine Kasuistik aussehen könnte. Eine solche ist aber auch ohne die-zumindest inhaltliche-Verknüpfung mit einem Leitprinzip schwer vorstellbar. Insofern schlägt die Unzulänglichkeit der Leitprinzipien auf die Kasuistik durch.

\section{b) Notwendigkeit einer strafschärfenden Abgrenzung}

Gleichzeitig ist auch eine Normgestaltung ohne strafschärfende Abgrenzung (in einem Einheitstatbestand oder einem privilegierungsbezogenen Zweistufensystem) ungenügend. Denn bei gleichbleibendem Erfolgsunwert (Tötung) kann das Handlungsunrecht auf subjektiver Seite (z.B. durch besondere Motive) und auf objektiver Seite (z.B. durch Art und Weise der Tatbegehung) erhöht sein. Solche Taten müssen hinreichend deutlich in der Norm aufgenommen werden, damit

46 Kritik bei: Eser (Fn. 12), 159; Lackner (Fn. 21), 36; Sotelsek (Fn. 7), 11.

47 Kritik bei: Müssig (Fn. 43), 116; Grünewald, Das vorsätzliche Tötungsdelikt, 2010, 48.

48 Kritik bei: Albrecht, Das Dilemma der Leitprinzipien auf der Tatbestandsseite des Mordparagraphen, JZ 1982, 697, 702; Sotelsek (Fn. 7), 34; vgl. Grünewald (Fn. 47), 52.

49 Kritik bei: Sotelsek (Fn. 7), 21; Eser (Fn. 12), 29.

50 Lackner (Fn. 21), 35; Staiger (Fn. 39), 188; Siol, Mordmerkmale in kriminologischer und kriminalpolitischer Sicht, 1973, 157.

51 Z.B. $\ 211$ Abs. 2 Nr. 2 und 3 des Vorschlags bei Eser (Fn. 12), 200 f.: Tötung mit Schusswaffe oder bandenmäßige Begehung; $\$ 211$ Abs. 2 Satz 1 Nr. 7 bei Lackner, Erfahrungen aus einem Seminar über die Reform der Strafvorschriften zum Schutze des Lebens, JZ 1977, 502, 503: Tötung, um auf andere Druck aufzubauen; $\mathbb{2} 211$ Abs. 2 Nr. 2 bei Reizel (Fn. 43), 240 f.: Tötung eines Hoheitsträgers.

52 Z.B. $\$ 211$ Abs. 2 Nr. 7 AE-Leben-Arbeitskreis AE, Alternativ-Entwurf Leben (AE-Leben), GA 2008,

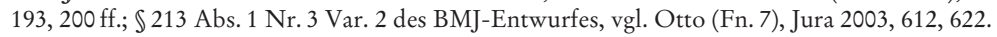

53 Kritik z.B. bei Albrecht (Fn, 48), JZ 1982, 697, 704. 
keine Probleme im Bereich der Bestimmtheit der Strafandrohung entstehen. ${ }^{54}$ Selbst wenn man keine Tatbestandsstufe annimmt, sind die Anforderungen an Präzision, Verlässlichkeit und Kontrollierbarkeit der Strafzumessungsregeln schon aufgrund des Strafrahmens, der von vermutlich fünf Jahren bis eventuell lebenslanger Haft geht, besonders hoch. ${ }^{55}$

\section{c) Unmöglichkeit einer perfekten Regelung und legislatives Zögern}

Eine trennscharfe und allseits befriedigende Unterscheidung zwischen Mord und Totschlag ist nicht möglich. ${ }^{56}$ Allen Arten und Kategorien bei den Reformvorschlägen stehen gewichtige Kritikpunkte entgegen, was die Reformwilligkeit des Gesetzgebers beeinflussen kann und vermutlich tut. Möglicherweise interpretiert die Legislative die beschriebenen Probleme dahingehend, dass in dem Licht der kritikwürdigen Reformvorhaben die aktuelle Gesetzesform zufriedenstellend wirkt. Diese Sicht wurde auch vereinzelt geäußert. ${ }^{57}{ }^{7} \mathrm{O} b$ das oft angemahnte Eingreifen des Gesetzgebers zu einem überzengenderen Ansatz [als die Rechtsfolgenlösung] fübren könnte, erscheint mindestens zweifelhaft. Vielleicht erklärt es sich auch so, dass grundlegende Reformbestrebungen im Bereich der $\iint 211 \mathrm{ff}$. $S t G B$ trotz einer Vielzabl von Appellen ausgeblieben sind. "58

Es ist grundsätzlich irrelevant, für welche Art der Reform der Gesetzgeber sich entscheidet, es wird immer fundierte Kritik geäußert werden (können). Da aber die Schwierigkeiten einer Reformierung bekannt sind, dürfte die Erwartungshaltung, die bei langem Warten steigen müsste, eher gering sein.

\section{Die Gefahr der Schaffung von Rechtsunsicherbeit}

Ein weiterer Grund für das Ausbleiben der Reform könnte sein, dass es durch neu entstehende Auslegungsprobleme zu einer "Jabre währenden Zeit der Unsicherbeit "59 kommen könnte. Dazu wird teilweise vermutet, dass der Gesetzgeber auch aus "mancherlei Rücksichtnabme auf Rechtsprechung "60 untätig bleibt. In der Tat könnten nach einer Reform manche jahrelang in der Rechtsprechung entwickelten Grundsätze hinfällig werden, und es würden neue Überlegungen nötig sein. Von daher ist es nicht verwunderlich, dass der Eindruck entstehen kann, dass in der Richterschaft einer grundsätzlichen gesetzlichen

54 Arbeitskreis AE (Fn. 52), GA 2008, 193, 219; Eser (Fn. 12), 95.

55 Vgl. BVerfG, NJW 2002, 1780. Für die Rechtsprechung stellen sich Abgrenzungsschwierigkeiten nicht weniger als für den Gesetzgeber. Deshalb offenbart sich der Fortschritt einer Bildung von „Fallgruppen“ durch die Rechtsprechung in einem Tatbestand ohne strafschärfende Abgrenzung nicht direkt; vgl. aber Deckers/Fischer/König/Bernsmann (Fn. 43), NStZ 2014, 9, 16; kritisch hierzu auch Krehl, Verfassungsrechtliche Grenzen einer Reform der Tötungsdelikte, ZRP 2014, 98.

56 Jähnke, Über die gerechte Ahndung vorsätzlicher Tötung und über das Mordmerkmal der Überlegung, MDR 1980, 705, 708; Frommel (Fn. 8), 76; vgl. BVerfGE 45, 187, 270; v. Liszt, in: Birkmeyer et al., Vergleichende Darstellung des deutschen und ausländischen Strafrechts BT V. Band, 1905, 68, schrieb über den Reformvorschlag von Carl Stooß: „Das Problem, zu einer neuen Begriffsbestimmung des Mordes zu gelangen, hat er nicht gelöst. Das ist kein Vorwurf: denn das Problem selbst ist unlösbar. “.

57 Wilms, Die Tötung mit gemeingefährlichen Mitteln, 2011, 269; Reichenbach, Die Rechtsfolgenlösung des BGH als Weg zur schuldangemessenen Strafe beim Mord, Jura 2009, 176, 177; Jescheck, in: Niederschriften über die Sitzungen der Großen Strafrechtskommission Bd. 7, 1959, 31: „Ich glaube, daß alle Lösungen, die ins Auge gefaßt wurden, ihre Mängel haben und daß es darum geht, jene zu finden, deren Mängel noch am geringsten erscheinen. Wenn man dies bedenkt, erscheint die Lösung des geltenden Rechts eigentlich ganz brauchbar.".

58 Reichenbach (Fn. 57), Jura 2009, 176, 183.

59 Fuhrmann (Fn. 44), 15.

60 Kreuzer, Über kriminologische Einmischung in Strafjustiz und Kriminalpolitik, in: Gropp/Lipp/Steiger (Hrsg.), Rechtswissenschaft im Wandel, 2007, 205 ff, 217. 
Neuregelung nicht nur vorbehaltlos positiv entgegengesehen wird. ${ }^{61}$ Allerdings sind grundlegende Reformen im Strafrecht oftmals sogar bei regem Widerstand der Praxis durchgeführt worden (z.B. Strafaussetzung zur Bewährung). ${ }^{62}$ Die Sorge vor zukünftiger Rechtsunsicherheit erscheint zudem nur dann nachvollziehbar, wenn eine solche aktuell nicht vorläge und somit eine negative Veränderung zu erwarten wäre. Ob aktuell große Rechtssicherheit besteht, ist aber durchaus fragwürdig. Auch durchläuft die Rechtsprechung immer wieder Veränderungen (z.B. bei Heimtücke). Zudem ist zu bedenken, dass eine qualitativ hochwertige Neuregelung die Konkretisierung durch die Rechtsprechung in einem viel geringeren Rahmen notwendig erscheinen lässt als dies aktuell der Fall ist. Insoweit könnte die Sorge vor einer Zeit von Grundsatzurteilen durchaus ein Hemmnis für Aktivitäten des Gesetzgebers darstellen; bei einer erfolgenden Reform ist eine jahrelange Rechtsunsicherheit aber nicht zu vermuten.

\section{Der Umfang einer Reform und der mit ibr verbundene Arbeitsaufwand}

Die Bundesregierung sah den Bedarf „einer umfassenden Auswertung der strafrechtlichen Rechtsprechung und vorhandenen wissenschaftlichen Erkenntnis$s e[n] " 63$ vor der Reform. Dabei kann zwar auf eine umfassende wissenschaftliche Durchdringung der komplexen und umfangreichen Materie zurückgegriffen werden. ${ }^{64}$ Aber die Rechtsprechung zu einzelnen Aspekten ist so umfangreich, dass eine umfassende Erfassung schwer möglich ist oder jedenfalls sehr lange dauern würde. Zudem existiert im Bereich von Mord und Totschlag ein „Publikationsexzeß“, der einen „Kollaps sinnvoller Strukturen" hervorruft. ${ }^{65}$

Auch sollen nach Angabe der Bundesregierung erhebliche Personalreduzierungen im Bundesministerium der Justiz für das Ausbleiben der Reform mitverantwortlich sein. ${ }^{66}$ Dies kann schwerlich überprüft werden. Zurückgegriffen werden kann dabei nur auf Angaben aus dem Handbuch der Justiz, in dem die im höheren Dienst tätigen Personen im Justizministerium aufgeführt werden. Um entscheidende Personalreduzierungen nachweisen zu können, müsste man zumindest die Anzahl der Juristen und der an der (Straf-)Gesetzgebung beteiligten Personen kennen. Es kann hier nur konstatiert werden, dass im höheren Dienst geringe Personalveränderungen auftraten, und insbesondere im Jahr 1996, als die entsprechende Aussage der Bundesregierung getätigt wurde, überdurchschnittlich viele Personen im Ministerium arbeiteten. Um eine Überlastung zu überprüfen, müssten aber auch Anzahl und insbesondere Umfang bearbeiteter Gesetzgebungsvorhaben beachtet werden, was hier nicht möglich ist.

\section{Die Reformvorschläge im Licht von Entwicklung und aktuellen Bestrebungen der Kriminalpolitik und Strafgesetzgebung}

Die lebenslange Freiheitsstrafe soll den hohen Rang des Rechtsguts Leben hervorheben. Eine Einschränkung dieser Sanktion könnte von der Gesellschaft als Einschränkung des Lebensschutzes verstanden werden. Geht der Gesetzgeber davon aus, eine Einschränkung würde von der Gesellschaft nicht akzeptiert werden, ist es nachvollziehbar, dass er die lebenslange Freiheitsstrafe nicht ein-

Vgl. Fuhrmann (Fn. 44), 7 (ehem. Richter am BGH) und Jähnke (Fn. 56), MDR 1980, 705, 708 (ehem. Vizepräsident des BGH); zu diesem Gedanken: Beckmann, Zur Neuregelung der vorsätzlichen Tötungsdelikte, GA 1981, 337, 341. 
schränken, geschweige denn abschaffen möchte. Dies zeigt sich auch am Arbeitsentwurf des Bundesjustizministeriums, worin an der Absolutheit der lebenslangen Strafe grundsätzlich festgehalten wurde. Es liegt also nicht fern, in Betracht zu ziehen, das Zögern des Gesetzgebers bei einer Befassung mit den vielfältigen Reformvorschlägen habe auch damit $\mathrm{zu}$ tun, dass diese fast ausnahmslos Einschränkungen in Bezug auf die Verhängung lebenslanger Freiheitsstrafen beinhalten. Für einen Gesetzgeber, der um symbolbehaftete Strafschärfungen - auch im hier relevanten Bereich ${ }^{67}$ - bemüht war und ist, ${ }^{68}$ ist die Einschränkung der lebenslangen Freiheitsstrafe schwierig. ${ }^{69}$ Da das Thema der Sanktion behandelt werden muss, wenn man eine Reform der Tötungsdelikte anstrebt, ist auch nachvollziehbar, warum keine kleineren Änderungen der Tatbestände vollzogen wurden. Somit behindert die Symbolik der lebenslangen Freiheitsstrafe eine emotionslose Diskussion, ${ }^{70}$ die das Zögern des Gesetzgebers in Bezug auf die Sanktion nachvollziehbar erscheinen lässt.

\section{Das Ausbleiben der Reform bei bestimmten Anlässen in den letzten 52 Jabren}

\section{Die früben Reformentwürfe (1962 und 1970)}

Schon Reformentwürfe, die in der Bundesrepublik sehr früh veröffentlicht wurden, enthielten Möglichkeiten der Umgestaltung der vorsätzlichen Tötungsdelikte. Damals konzentrierte man sich jedoch in Gesetzgebung und Wissenschaft auf Veränderungen im Allgemeinen Teil des Strafgesetzbuches. ${ }^{71}$ Im Besonderen Teil ging es mehr darum, ob bestimmte Verhaltensweisen strafbar sein sollen. ${ }^{72}$ Die Reform vorsätzlicher Tötungsdelikte ist schon deshalb zu diesem Zeitpunkt nicht erfolgt. ${ }^{73}$

67 Vgl. zu der nur vermeintlichen „doppelten Sicherung“ durch die seit 2002 bestehende Möglichkeit, Sicherungsverwahrung neben lebenslanger Freiheitsstrafe anzuordnen, Kreuzer, Notwendigkeit der Reform des Tötungsstrafrechts und der „AE-Leben“, in: Dölling (Hrsg.), Festschr.f. Schöch, 2010, $495 \mathrm{ff} ., 502$.

68 Frommel, 40 Jahre Strafrechtsreform, NK 2008, 133, 135; Hassemer, Das Symbolische am symbolischen Strafrecht, in: Achenbach et al. (Hrsg.), Festschr.f. Roxin, 2001, $1001 \mathrm{ff} ., 1007 \mathrm{f}$. Zuletzt wurde durch den mit dem Gesetz zur Erweiterung der jugendgerichtlichen Handlungsmöglichkeiten vom 4.9.2012 (BGBl. I, 1854) eingeführten $\$ 105$ Abs. 3 Satz 2 JGG die Möglichkeit geschaffen, bei von einem Heranwachsenden begangenen Mord unter bestimmten Voraussetzungen nicht nur 10, sondern nunmehr bis zu 15 Jahre Jugendstrafe zu verhängen.

69 Rotthaus, Ergänzende Erhebungen über die Verhängung und die vorzeitige Beendigung der lebenslangen Freiheitsstrafe, NStZ 2005, 482; Kreuzer (Fn. 60), 217.

70 Müssig (Fn. 43), 1; Linka (Fn. 9), 253.

71 Hirsch, Der Umgang des Gesetzgebers mit dem StGB und die Notwendigkeit der gesetzgeberischen Berichtigung unterlaufener gesetzestechnischer Fehler, in: Paeffgen et al. (Hrsg.), Festschr.f. Puppe, 2011, $105 \mathrm{ff} ., 108$.

72 Z.B. Straftaten gegen die Sittenordnung, Abtreibung, vgl. Ostman von der Leye, Zur Reform des Besonderen Teils des Strafgesetzbuches, 1968, 61 ff.; auch das politische Strafrecht machte einen Großteil der Beschäftigung mit dem Besonderen Teil aus, vgl. Worm, SPD und Strafrechtsreform, 1968, $116 \mathrm{f}$.

73 Der E 1962 (BT-Drucks. IV/650), der eine Neuregelung von Mord und Totschlag vorsah, wurde Anfang der 5. Wahlperiode erneut dem Bundestag zur Entscheidung vorgelegt (BT-Drucks. V/32). Der AE 1966, der zu den vorsätzlichen Tötungsdelikten schwieg, wurde auch im Bundestag eingebracht (BTDrucks. V/2285). Beide wurden dem Sonderausschuss für die Strafrechtsreform überwiesen. Dieser ebnete 1969 mit seinem Ersten (BT-Drucks. V/4094) und Zweiten Schriftlichen Bericht (BT-Drucks. V/4095) den ersten beiden Strafrechtsreformgesetzen den Weg. Bis auf den kurzen Hinweis, dass man sich auf die wichtigsten Neuregelungen zu beschränken habe (BT-Drucks. V/4094, 2), finden sich keine Ausführungen dazu, weshalb der Reformvorschlag (E 1962) in Bezug auf vorsätzliche Tötungsdelikte unberücksichtigt blieb. 
Das Gutachten von Eser zeigte erschreckend deutlich auf, wie schwierig eine Neustrukturierung der vorsätzlichen Tötungsdelikte ist. Mit dem Votieren für eine privilegierungsbezogene zweistufige Gefährlichkeitslösung ${ }^{74}$ ging man einen Weg, der viele folgende Reformvorschläge prägte. Die Abkehr von einem Verwerflichkeitskonzept und der damit einhergehende Wegfall der Motivgeneralklausel musste aber bei einem „punitiven Gesetzgeber“ Bedenken auslösen. Zudem war der Gesetzgeber durch das Urteil von 1977 zur Verfassungsmäßigkeit der lebenslangen Freiheitsstrafe ${ }^{75}$ in seiner abgrenzungsbezogenen Untätigkeit gestärkt worden. Die Einführung des $₫ 57$ a StGB im Jahr 1981 relativierte objektiv die Abgrenzungsprobleme durch die Möglichkeit einer Unterscheidung auf Vollstreckungsebene und wirkte sich damit vermutlich auch auf weiteres legislatives Handeln negativ aus.

\section{Das 6. StrRG (1998) und der Arbeitsentwurf des Bundesjustizministeriums (2001)}

Die erste große Reform des Besonderen Teils (6. StrRG) erfolgte in einem Zeitraum von nur 18 Monaten; in einer derart kurzen Zeitspanne war eine Reform der hier gegenständlichen Art nicht möglich. ${ }^{76}$ Weshalb der spätere Arbeitsentwurf des Bundesjustizministeriums nicht umgesetzt wurde, ist nicht bekannt. Inhaltlich könnte die eher extensive Kodifizierung der restriktiv anzuwendenden Rechtsfolgenlösung ${ }^{77}$ in $\ 212$ Abs. 2 E BMJ dem „punitiven Gesetzgeber“ eine zu starke Einschränkung lebenslanger Freiheitsstrafe bei heimtückischen Tötungen gewesen sein. ${ }^{78}$ Dies wäre aber leicht zu korrigieren gewesen. Anderweitige Einschränkungen der lebenslangen Freiheitsstrafe waren mit dem Entwurf gerade nicht verbunden. Eventuell war auch das Ende der Legislaturperiode verbunden mit dem Wechsel der Ministerposition - im Oktober 2002 mitverantwortlich dafür, dass der Arbeitsentwurf scheinbar nicht weiter verfolgt wurde. Weitergehende Anhaltspunkte für Gründe des Ausbleibens der Reform liegen nicht vor.

\section{Die mögliche Gestaltung einer Reform}

Die Befassung mit der Frage, wie eine Reform der vorsätzlichen Tötungsdelikte ausfallen könnte, kann in rechtliche und tatsächliche Aspekte unterteilt werden. Die rechtlichen Überlegungen sind dabei hinreichend bekannt. Da bei den Reformbemühungen Probleme offenbart und aktiv angegangen werden, ist es möglich, de lege ferenda „bessere“ Normen zu formulieren: So hat die Entscheidung für ein Leitprinzip trotz der erkannten Mängel schon deutliche Vorteile im Vergleich zum Mischen von bis zu drei (Leit-)Prinzipien de lege lata. Auch bessere Formulierungen im Rahmen einer Kasuistik zu finden, erscheint durchaus möglich, da die qualifizierenden Merkmale aktuell erst durch die ergebnisorientierte

76 Linka (Fn. 9), 249; vgl. Sander/Hohmann, Sechstes Gesetz zur Reform des Strafrechts (6. StrRG), NStZ $1998,273$. 
Auslegung der Rechtsprechung erträglich werden. Eine umfangreiche eigene Positionierung diesbezüglich ist im gegebenen Rahmen nicht möglich. ${ }^{79}$

\section{Konzentration auf notwendige Änderungen}

Die lebenslange Freiheitsstrafe kann bei einer Reform nicht weiter in ihrer Absolutheit bestehen bleiben, weil dies ein Grundübel der geltenden Gesetzeslage darstellt. Eine Abschaffung dieser Strafandrohung erscheint derzeit leider unrealistisch. Zumindest würde die Gefahr bestehen, dass über die emotionsgeladene Diskussion um die lebenslange Freiheitsstrafe abgrenzungsbezogene Aspekte zu wenig Beachtung finden und eine Reform daran scheitert. Die lebenslange Freiheitsstrafe könnte aber zumindest eingeschränkt werden, etwa durch alternative Androhung, Typenkorrektur oder die Normierung von Milderungsgründen, die die Höchststrafe ausschließen. Insofern erscheint es zwar kaum wünschenswert, die lebenslange Freiheitsstrafe formal aufrecht zu erhalten, dies erhöht aber vermutlich die Durchführungswahrscheinlichkeit der Reform. ${ }^{80} \mathrm{Je}$ mehr man die lebenslange Freiheitsstrafe einschränkt oder sogar abschafft, desto eher müssten auch bei anderen Straftatbeständen die Sanktionen im Verhältnis zu den Fällen schwerster Tötungen überdacht werden, ${ }^{81}$ was wiederum den Umfang einer Reform drastisch ausweiten würde. Neben der Abschaffung lebenslanger Freiheitsstrafe sollten auch Aspekte der Sterbehilfe ausgeklammert werden. ${ }^{82}$ Auch die Dringlichkeit einer Reform von $\ 57 \mathrm{a} \mathrm{StGB}^{83}$ sollte vorher überdacht werden.

Gleichzeitig erscheint die teilweise geforderte sprachliche Neufassung, ${ }^{84}$ also das Ersetzen der Worte Mörder und Totschläger, was unproblematisch möglich ist ${ }^{85}$ aus zwei Gründen nicht sinnvoll: Einerseits würde man bis auf die WortlautThematik kein weiteres Problem lösen. Erwähnenswert ist in diesem Zusammenhang ein Versanden der Reformbestrebung des Bundesjustizministeriums im Jahre 2001, die als eine hauptsächliche Veränderung das Entfernen der Formulierung „Mörder ist“ aufwies. Hieraus lässt sich eine mögliche Sichtweise des Gesetzgebers ableiten, wonach die Formulierung nicht besonders schlimm sei, weil die von den Nationalsozialisten geprägte Tätertypenlehre in der Praxis nicht mehr angewandt wird, 86 die Formulierung „Mörder ist“ sich inhaltlich nicht mehr von der Formulierung „Mord begeht“ unterscheidet ${ }^{87}$ und das Maß des

79 Ohne näher inhaltlich darauf eingehen zu können, erscheint der vergleichsweise aktuelle AlternativEntwurf Leben (Arbeitskreis AE [Fn. 52], GA 2008, 193, 200 ff.) viele erfreuliche Regelungsperspektiven aufzuzeigen. Dieser Entwurf verdient auch besondere Beachtung, weil er bei dem zweiten Strafrechtsdialog in Karlsruhe ,sehr positiv “ aufgenommen wurde, vgl. Roxin, Auch Praktiker sind Wissenschaftler, in: Jahn/Nack (Hrsg.), Rechtsprechung, Gesetzgebung, Lehre, 2010, 101 ff., 102.

80 So deutet auch Krehl (Fn. 55), ZRP 2014, 98 die aktuellen Äußerungen des Bundesjustizministers. Dieser stellte in einer Überschrift die Frage „Große Koalition = Große Reform?"“, Maas (Fn. 3), RuP 2014, 65, 67. Dem darunter gefassten Text lässt sich dann nicht entnehmen, dass die Frage positiv zu beantworten ist; die lebenslange Freiheitsstrafe solle gerade nicht in Frage gestellt werden.

81 Hirsch (Fn. 10), 230.

82 Vgl. Hirsch, Bilanz der Strafrechtsreform, in: Hirsch/Kaiser/Marquardt (Hrsg.), Gedächtnisschr.f. Kaufmann, 1986, 133 ff., 155.

83 Für eine Abschaffung beispielsweise Deckers/Fischer/König/Bernsmann (Fn. 43), NStZ 2014, 9, 16.

84 Siehe BR-Drucks. 54/14, Gesetzantrag des Landes Schleswig-Holstein: Entwurf eines ... Gesetzes zur Änderung des Strafgesetzbuches - Bereinigung der $\$ \mathbb{S} 211 \mathrm{ff}$. (... StRÄndG); vgl. Spoorendonk (Fn. 2), SchlHA 2014, 77, 78.

85 Hirsch (Fn. 71), 116; Wolf, Mörder oder Totschläger? Zum 60jährigen Bestehen des $\$ 211$, in: Amelung et al. (Hrsg.), Festschr.f. Schreiber, 2003, 519 ff., 532; Köhne, Immer noch reformbedürftig: Strafvorschriften zur vorsätzlichen Tötung, ZRP 2014, 21, $21 \mathrm{f}$.

86 Die Bedeutungslosigkeit für die aktuelle Rechtsanwendung anerkennend auch Spoorendonk, Die Lehre vom Tätertyp. Ist das Strafrecht NS-belastet? Pro: NS-Rechtserneuerer wollen ein gesinnungsethisches Täterstrafrecht, RuP 2014, 42.

87 Arvanitis, Zur Problematik der Unterscheidung zwischen Mord und Totschlag, 1982, 57. 
Verbleibs nationalsozialistischen Gedankenguts in den Normen als sehr gering anzusehen ist. ${ }^{88}$ Diese These wird durch die Antwort der Bundesregierung auf die diesjährige Kleine Anfrage zur Notwendigkeit der sprachlichen Bereinigung der $\int S 211$ und 212 StGB gestützt. ${ }^{89}$ Andererseits würde man sich durch eine erfolgende (kleine) Reform gleichzeitig eines der wenigen medienwirksamen Argumente berauben: Würde durch eine Reform zur Befriedung der Öffentlichkeit tatsächlich nur das Entfernen der Symbole der Tätertypenlehre bewirkt, gäbe es noch weniger Anreiz für einen Gesetzgeber, die Materie (erneut) aufzugreifen. Es gilt hier zu verhindern, dass eine Reform erfolgt, die durch ihre Konzentration momentan leicht geöffnete Türen für immer schließt.

\section{Das Zusammenspiel von Wissenschaft und Politik}

In der von Bundesjustizminister Maas am 20. Mai 2014 einberufenen Expertengruppe zur Überarbeitung der Tötungsdelikte befinden sich neben vielen Praktikern auch einige Wissenschaftler. ${ }^{90}$ Die nunmehrige Beteiligung der Wissenschaft am Reformprozess ist als sehr wichtig und erfreulich einzustufen, da die bisherigen Leistungen der Wissenschaft von der Politik nicht umfassend gewürdigt wurden. Für den Gesetzgeber hat dies zudem die positive Auswirkung, dass am Gesetzgebungsprozess beteiligte Wissenschaftler eher bereit sind, die endgültige Norm auch im Schrifttum zu verteidigen. ${ }^{91}$ Gleichzeitig kann so das Risiko, dass der Gesetzgeber für die Unmöglichkeit der perfekten Reform „haftbar" gemacht wird, ${ }^{92}$ herabgesenkt werden.

Auch wenn nun eine Auswahl an Wissenschaftlern am Reformprozess beteiligt ist, sollten andere Wissenschaftler weiterhin versuchen, direkt Einfluss auszuüben und den tendenziell eher schwachen Kontakt zur Politik zu stärken. Dabei ist es wichtig, schon möglichst früh auf die Grundrichtung der Reform Einfluss zu nehmen. Ein Blick auf die Geschichte zeigt, dass dies möglich ist. Für die ersten Strafrechtsreformgesetze war der Alternativ-Entwurf von $1966^{93}$ von großer Bedeutung. ${ }^{94}$ Dort war es gelungen, den Prozess der Gesetzgebung in hohem Maße zu beeinflussen. ${ }^{95}$ Dies erreichte man hauptsächlich durch die Verpflichtung jedes der sechzehn Mitverfasser, sich zweimal im Monat zu den Themen in den Medien zu äußern, wodurch eine öffentliche Debatte fundiert stattfinden konnte. Zudem wandte man sich schon 1967 mit der Bitte um Berücksichtigung des Entwurfes im Wege eines „Rundschreiben[s] an die Mitglieder des Deutschen Bundestages“ gezielt an die Legislative. Auch bei der Veröffentlichung des Entwurfs gelang es, durch synoptische Darstellung der lex lata und des Entwurfes mit kurzen und verständlichen Begründungen das Anliegen auch juristischen Laien verständlich zu machen. Hinzu kam die Eignung des Entwurfs, als Bundestags-

91 Arzt, Wissenschaftsbedarf nach dem 6. StrRG, ZStW 1999, 757, 765.

92 Die Sorge davor könnte auch ein Grund für das Ausbleiben der Reform sein, vgl. Müssig (Fn. 43), 1; Linka (Fn. 9), 253.

93 Vgl. dazu Fn. 73.

94 Baumann, Lehren aus einer Strafrechtsreform, Universitas 1983, 17, 19.

95 Stächelin, Strafgesetzgebung im Verfassungsstaat $1998,327$. 
drucksache in die parlamentarischen Beratungen übernommen zu werden. ${ }^{96}$ Die Parameter des Alternativ-Entwurfs 1966 lassen sich nicht kopieren. Die Leidenschaft damaliger Reformbemühungen war jedoch beispielhaft.

\section{Die Einbeziehung der Öffentlichkeit}

Durch den Vorstoß der schleswig-holsteinischen Justizministerin Anke Spoorendonk bei der Justizministerkonferenz am 14.11.2013 in Berlin zur Notwendigkeit einer Reform aufgrund der nationalsozialistischen Herkunft ${ }^{97}$ gelangte die Thematik wieder ${ }^{98}$ auch in die nicht-juristische Presse und wurde einem breiteren Publikum zugänglich gemacht. ${ }^{99}$ Die Einbeziehung der Öffentlichkeit muss im Reformprozess eine große Rolle spielen.

Denn damit eine Reform, bei der die lebenslange Freiheitsstrafe eingeschränkt wird, von der Gesellschaft angenommen wird, muss diese informiert werden. Insbesondere ist zu verdeutlichen, dass eine objektive Einschränkung der lebenslangen Freiheitsstrafe und (eventuell) ein Verzicht auf eine tatbestandliche Abgrenzung schwerer Tötungen keine Einschränkung des Lebensschutzes bedeuten. Vor allem in diesem Bereich kann man damit rechnen, dass die Medien aus Eigenantrieb eher Ängste schüren wollen, ${ }^{100}$ sodass ein Gegensteuern notwendig sein wird. Die öffentliche Meinung in Bezug auf Tötungsdelikte und das "Sicherbeitsbedürnis der Bevölkerung "101 wird der Gesetzgeber in seine Reformpläne einbeziehen, damit etwaige Veränderungen eine möglichst hohe Achtung erfahren. Dies könnte unter anderem dafür sprechen, den Begriff Mord für besonders schwerwiegende Tötungen beizubehalten. Würde man jedoch im Rahmen der Reform ein Modell wählen, bei dem auf eine Tatbestandsstufe zu qualifizierten Tötungen verzichtet wird, würden auch „Durchschnittstötungen“ als Mord bezeichnet werden. Ein Strafrahmen, der nunmehr z.B. bei fünf Jahren beginnt, könnte dann den Eindruck erwecken, dass „Mord“ in Zukunft weniger streng geahndet werde. Bei Beibehalten des Begriffes Mord erscheint mithin eine Tatbestandsstufe zu qualifizierten Tötungen - unter Reformförderungsaspekten - sinnvoll. ${ }^{102}$

Zudem ist es aufgrund von - eventuell tiefgreifenden - Veränderungen in einem hochsensiblen Bereich des Strafrechts wichtig, den Bürgern die Notwendigkeit der Reform deutlich zu machen, damit die Reform nicht aufgrund einer negativen Beeinflussung des Gesetzgebers von der mangelnden Reformbereitschaft in der Gesellschaft ausbleibt. Aus dieser Perspektive ist es erfreulich, dass der Bundesjustizminister über die Aussagen zur nationalsozialistischen Herkunft hinausgeht, mit der aktuellen Benachteiligung der Frauen durch das Mordmerkmal

Vgl. Stächelin (Fn. 95), 328; Scheffler, Das Reformzeitalter 1953-1975, in: Vormbaum/Welp (Hrsg.), Das Strafgesetzbuch - Sammlung der Änderungsgesetze und Neubekanntmachungen - Supplementband 1, 2004, 174 ff., 197.

Vgl. den Beschluss der Justizministerkonferenz, http://www.saarland.de/dokumente/res_justiz/ TOP_II.8-Bereinigung_der_211_ff_StGB.pdf (abgerufen am 20.6.2014).

Kreuzer, FAZ 5.6.2008, 8, gelang es beispielsweise im Rahmen der medialen Aufmerksamkeit um den Kannibalen von Rotenburg, ausführlich auf die Reformbedürftigkeit der Tötungsdelikte hinzuweisen. Fischer, Die Zeit Nr. 51, 12.12.2013, 8; Prantl, SZ Nr. 295, 21.12.2013, 1; vgl. auch Spoorendonk (Fn. 2), SchlHA 2014, 77, 78. Rotthaus (Fn. 69), NStZ 2005, 482.

Vgl. hierzu die Rede des Bundesjustizministers zum Auftakt der Expertengruppe „Überarbeitung der Tötungsdelikte“, http://www.bmjv.de/SharedDocs/Reden/DE/2014/20140520_Expertengruppe_Toetungsdelikte.html?nn=1468700 (abgerufen am 20.6.2014).

Man würde damit auch Diskussionen über die Verjährung von Mord ( $\mathbb{} 78 \mathrm{Abs}$. $2 \mathrm{StGB}$ ) ausklammern und Reformpläne entschlacken können. Mitsch, Konsequenzen der Abschaffung des $\$ 211$ StGB, StV 2014, 366, 369, geht davon aus, dass an Veränderungen des Verjährungsrechts eine Reform nicht scheitern würde. 
Heimtücke ein medienwirksames und pointiertes Beispiel für die Notwendigkeit der Reform nutzt und das Reformbedürfnis somit massengerecht aufbereitet. ${ }^{103}$

\section{Schlussbemerkung}

Es liegen diverse Gründe vor, weshalb der Gesetzgeber die vorsätzlichen Tötungsdelikte, insbesondere in Bezug auf die Abgrenzung von Mord und Totschlag, bisher nicht reformiert hat. Ist man sich dieser Gründe bewusst, kann man ihnen gezielt entgegenwirken. Eine gesetzliche Regelung, die rechtsstaatlichen Maßstäben mehr genügt als die lex lata und die Rechtssicherheit und Einzelfallgerechtigkeit fördert, kann gefunden werden.

Das Anpacken einer Reform der vorsätzlichen Tötungsdelikte ist des Schweißes der Edlen wert. Die Notwendigkeit einer Reform ist deutlich, die Hemmnisse sind erkennbar, Lösungsmöglichkeiten sind diskutiert. Vieles ist überlegt, geschrieben und überdacht worden. Das alles darf nicht versanden. Wenn jetzt die Kräfte zu einem gemeinsamen Ziel gebündelt würden, könnte es gelingen, dass ein wichtiger Bereich des Strafrechts die akzeptable und möglichst sogar zufriedenstellende Lösung erfährt, auf die so lange vergeblich gewartet werden musste.

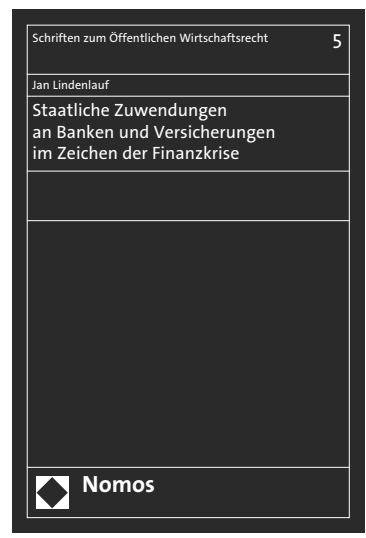

Staatliche Zuwendungen an Banken und Versicherungen im Zeichen der Finanzkrise

Von Dr. Jan Lindenlauf

2014, 261 S., brosch., 68,-€

ISBN 978-3-8487-1430-8

(Schriften zum Öffentlichen

Wirtschaftsrecht, Bd. 5)

www.nomos-shop.de/22749

Das Werk widmet sich der beihilferechtlichen Bewertung von staatlichen Zuwendungen an Banken und Versicherungen und untersucht, inwieweit eine Ausweitung der Gesetzgebung zur Finanzmarktstabilisierung auf Versicherungen zielführend ist. Zusätzlich werden wesentliche Aspekte der Finanzkrise und die Reaktionen der Europäischen Kommission erläutert.

Portofreie Buch-Bestellungen unter www.nomos-shop.de

Alle Preise inkl. Mehrwertsteuer

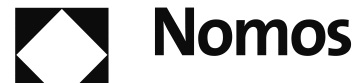

